\title{
Clinical Characteristics and Service Needs of Alternate-Level-of-Care Patients Waiting for Long-Term Care in Ontario Hospitals
}

Caractéristiques cliniques et besoins en services pour les patients nécessitant d'autres niveaux de soins et inscrits sur les listes d'attente pour les hôpitaux de soins de longue durée en Ontario

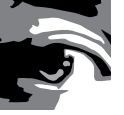

by ANDREW P. COSTA, BSC(HON)

Research Associate, Department of Health Studies and Gerontology

University of Waterloo

Waterloo, ON

JOHN P. HIRDES, PHD

Ontario Home Care Research and Knowledge Exchange Chair Professor, Department of Health Studies and Gerontology

University of Waterloo

Waterloo, ON

Scientific Director, Homewood Research Institute

Guelph, ON 


\section{Clinical Characteristics and Service Needs of Alternate-Level-of-Care \\ Patients Waiting for Long-Term Care in Ontario Hospitals}

\section{Abstract}

Background: Alternate-level-of-care (ALC) patients waiting for admission to longterm care (LTC) in Ontario hospitals are an important subgroup of ALC patients. Understanding the characteristics of ALC patients waiting for LTC can identify opportunities for alternative discharge options.

Methods: Characteristics from a sample of 13,915 ALC patients waiting for LTC in acute and complex hospitals were analyzed and compared to those from a sample of 113,046 long-stay home care clients in Ontario. Population-level data were obtained using Ontario's RAI-HC database for the period January 2007 to September 2008. Findings: Relative to older long-stay home care clients, ALC patients waiting for LTC admission have more functional impairment and complex health needs. A combination of targeted transitional care and more resource-intensive home care services are needed to successfully address ALC patients' care needs in the community.

Conclusions: A substantial portion of ALC patients waiting for LTC placement in acute and complex care hospitals may be more appropriately cared for in community settings if adequate community-centred resources are put in place.

\section{Résumé}

Contexte: Les patients nécessitant d'autres niveaux de soins (ANS) qui attendent d'être admis dans un hôpital de soins de longue durée (SLD) en Ontario constituent un sous-groupe important de tous les patients ANS. Mieux connaître les caractéristiques des patients ANS inscrits sur ces listes d'attente peut aider à dégager diverses options pour les sorties d'hôpital.

Méthodologie : Nous avons analysé les caractéristiques d'un échantillon de 13915 patients ANS en milieu hospitalier de courte durée ou de soins complexes et inscrits sur les listes d'attente pour les hôpitaux de SLD, et nous avons comparé ces données avec celles d'un échantillon de 113046 clients de soins prolongés à domicile, en Ontario. La base de données RAI-HC de l'Ontario a servi à obtenir des données populationnelles, entre janvier 2007 et septembre 2008.

Résultats : Comparativement aux clients plus âgés qui reçoivent des soins prolongés à domicile, les patients ANS qui attendent d'être admis dans un établissement de SLD présentent davantage de déficience fonctionnelle et de besoins en services de santé complexes. Il faudrait concevoir une combinaison de soins de transition ciblés et de services de soins à domicile plus axés sur les ressources, afin de traiter adéquatement les besoins en services des patients ANS dans la communauté.

Conclusions: Une grande partie des patients ANS, en milieu hospitalier de courte durée ou de soins complexes et inscrits sur les listes d'attente pour être admis dans un établissement de SLD, pourraient être traités plus adéquatement dans des établissements communautaires, si des ressources axées sur la communauté sont mises en place. 
$\mathrm{E}$ FFECTIVE MANAGEMENT OF THE NEEDS OF OLDER ADULTS IN ACUTE HOSPItals is a key challenge for healthcare systems in Canada and internationally. Of particular concern is the large number of hospital beds being occupied by older patients who no longer need acute hospital services, but have not been discharged because of ongoing post-acute care needs or inadequate supports in the community. In Ontario, these individuals are commonly designated as alternate-level-of-care (ALC) patients. ALC days in Ontario's acute hospitals have been increasing since 1995, and by 2003, ALC patients accounted for approximately $10 \%$ of all acute care beds (OACCAC et al. 2006). Since then, the proportion of ALC patients has almost doubled, comprising 19\% of all current acute care beds in Ontario (Ontario Hospital Association [OHA] ALC Survey Results 2009).

The consequences of the growing numbers of ALC patients for hospitals and the healthcare system have been widely reported (ALC Expert Panel 2006; CIHI 2009; OACCAC et al. 2006; Rock et al. 1995). However, it is generally acknowledged that ALC patients are not the cause of patient flow inefficiencies within the healthcare system. Rather, the growing number of ALC patients reflects a failure of the healthcare system to meet the needs of older adults with complex and declining health (ALC Expert Panel 2006). Although there has been much discussion about the "ALC challenge," less attention has been paid to the needs and experiences of ALC patients. Prolonged hospitalization is associated with the incidence of adverse outcomes, including accelerated functional decline, delirium, pressure ulcers, infections and falls (Covinsky et al. 2003; Creditor 1993; Graf 2006: Lim et al. 2006). For example, there is evidence that between $30 \%$ and $60 \%$ of older persons develop new ADL functional dependencies during their hospital stay (Fortinsky et al. 1999; Sager et al. 1996).

Patients waiting for long-term care placement in hospitals are an important subgroup within the ALC patient population. In Canada, the longest median ALC stays in acute hospitals belonged to those waiting for LTC, with a median stay of 15 days, compared to the 10-day national average and the 6-day average for those waiting for a rehabilitation bed (CIHI 2009). In addition, ALC patients waiting for LTC accounted for $60 \%$ of all ALC days in Canada (provincial range: 49\%-76\%) (CIHI 2009). In Ontario, $55 \%$ of acute ALC patients and $80 \%$ of complex continuing care (CCC) ALC patients are waiting for admission to LTC (OHA ALC Survey Results 2009).

Opportunities to make better use of community care services are being explored in Canada, with the aim to redirect some ALC patients waiting for LTC back into the community. For example, the Alberta Continuing Care Strategy, among other objectives, hopes to provide assistance and funding for some individuals to move back into their communities (Government of Alberta 2008). Prince Edward Island's Healthy Aging Strategy includes "transitional care" as one of its pillars (Department of Health 
and Wellness 2009). Also, Ontario's provincial ER/ALC and Aging at Home strategies both attempt to foster expanded home care/community supports to avoid or delay admission to LTC (MOHLTC 2008, 2009). Although many healthcare initiatives target ALC as a priority, relatively little is known about the demographic and clinical characteristics of ALC patients and how they compare to existing community care populations. Understanding the characteristics of ALC patients provides a starting point for optimal capacity planning and the pursuit of discharge options appropriate to ALC patients' strengths, preferences and needs.

The objective of this study was to compare ALC patients waiting for LTC in acute and complex hospitals to long-stay home care clients using RAI-Home Care (RAIHC) assessments completed by Ontario Community Care Access Centre (CCAC) case managers.

\section{Methods}

Sample

This investigation utilized a prevalence sample of 13,915 ALC patients waiting for LTC admission and 113,046 age 65 or older long-stay home care clients assessed with the RAI-HC in Ontario from January 2007 to September 2008. The most recent, unique RAI-HC assessment occurring during the sample period was included in this analysis to control for multiple assessment bias within groups. However, a small portion of the sample (3.6\%) were hospital-assessed patients and then subsequently reassessed in the community. Ethics clearance was given for secondary use of these data by the University of Waterloo Office of Research Ethics (ORE\#14761).

\section{Data}

The interRAI family of assessment instruments and their related applications comprise an integrated health information system that captures important health and service utilization domains of individuals requiring care (Gray et al. 2009; Hirdes et al. 1999, 2008a). Since 2004, all Ontario ALC patients waiting for a LTC bed in acute and complex hospitals, and who are not expected to go home, receive the hospital version of the RAI-HC assessment to initiate their LTC application process. In addition, CCAC case managers have used the community version of the RAI-HC since 2002 to assess all long-stay home care clients who are expected to receive services longer than 60 days. ALC patients are identifiable in the RAI-HC database because they were the group that received the hospital version of the assessment. The Ontario RAIHC database currently includes over 60,000 records of ALC patients assessed in acute and complex hospitals while awaiting potential placement into LTC, and over 1 million records for long-stay home care clients. 
The RAI-HC is a comprehensive assessment of a person's strengths, preferences and needs. As one of four interRAI instruments mandated for use in Ontario, the RAI-HC reliably documents important domains of a person's well-being, including health, function, social support, service use and quality of life (Morris et al. 1997; Poss et al. 2008). Subsets of these items are used to generate summary scales, including the Cognitive Performance Scale (CPS) (Morris et al. 1994), the Depression Rating Scale (DRS) (Burrows et al. 2000; Koehler et al. 2005; Martin et al. 2008), the Changes in Health, End-stage Disease, and Signs and Symptoms (CHESS) Scale (Hirdes et al. 2003) and the Method for Assigning Priority Levels (MAPLe) (Hirdes et al. 2008b). The RAI-HC includes care-planning protocols, referred to as clinical assessment protocols (CAPs). These CAPs were created for RAI/MDS/interRAI instruments in use for community health, home care, assisted living and LTC. CAPs are automatically generated from the assessment items to provide a comprehensive risk and needs assessment as well as to aid the provision of evidence-based services (CIHI 2009). Individual CAPs can be triggered for resolving problems, reducing the risk of decline or increasing the potential for improvement.

\section{Analysis}

Descriptive analysis was performed using SAS Version 9.1 for Windows (SAS Institute, Cary, NC). All confidence intervals were calculated at the $99 \%$ level (alpha $=0.01)$. Confidence intervals listed for each proportion were based on the standard error of a proportion. The majority of comparisons between groups achieved significance given the large sample sizes involved. Although statistical significance is necessary for clinical significance, it may not be sufficient to warrant a change in policy or service practice. Discretion should be exercised when considering actions needed in response to comparisons where the difference between groups is significant, but small.

\section{Results}

As shown in Table 1, the majority of ALC patients waiting for LTC were over age 75 $(80.0 \%, 99 \%$ CI: 79.1, 80.9) (and close to half were over age 85$)$. ALC patients waiting for LTC placement were more likely to be male, unmarried and living alone, compared to home care clients. The majority of ALC patients waiting for LTC previously lived in a private residence (79.5\%, 99\% CI: 78.6, 80.4), and the majority of these did not access home care service(s) through a CCAC. A modest proportion of these ALC patients previously resided in a LTC facility $(12.7 \%, 99 \%$ CI: $12,13.4)$. 
Clinical Characteristics and Service Needs of Alternate-Level-of-Care

Patients Waiting for Long-Term Care in Ontario Hospitals

TABLE 1. Demographic characteristics, primary caregiver characteristics and acute hospital utilization

\begin{tabular}{|c|c|c|c|c|c|c|c|c|c|}
\hline & & \multicolumn{2}{|c|}{$\begin{array}{l}\text { Home care } \\
\text { ( } \geq 65 \text { years) }\end{array}$} & \multicolumn{2}{|c|}{$\begin{array}{l}\text { ALC } \\
\text { (AII) }\end{array}$} & \multicolumn{2}{|c|}{$\begin{array}{c}\text { ALC } \\
(<75 \text { years })\end{array}$} & \multicolumn{2}{|c|}{$\begin{array}{c}\text { ALC } \\
\text { ( } \geq 75 \text { years) }\end{array}$} \\
\hline & & $\%(\mathrm{Cl})$ & $\mathbf{N}$ & $\%(C l)$ & $\mathbf{N}$ & $\%(\mathrm{Cl})$ & $\mathbf{N}$ & $\%(C l)$ & $\mathbf{N}$ \\
\hline \multicolumn{10}{|c|}{ Demographic Characteristics } \\
\hline \multicolumn{2}{|l|}{ Age (mean) } & $\begin{array}{c}82.1 \\
( \pm .06)\end{array}$ & 113,046 & $\begin{array}{c}83.0 \\
( \pm 0.1)\end{array}$ & 13,915 & $\begin{array}{c}70.2 \\
( \pm 0.2)\end{array}$ & $|, 84|$ & $\begin{array}{c}85.4 \\
( \pm 0.1)\end{array}$ & 12,074 \\
\hline Gender & Female & $\begin{array}{c}68.0 \\
( \pm 0.4)\end{array}$ & 76,812 & $\begin{array}{c}61.5 \\
( \pm 0.1)\end{array}$ & 8,537 & $\begin{array}{c}51.6 \\
( \pm 3.0)\end{array}$ & 949 & $\begin{array}{c}62.9 \\
( \pm 1.1)\end{array}$ & 7,588 \\
\hline \multirow{2}{*}{ Marital status } & Married & $\begin{array}{c}37.3 \\
( \pm 0.4)\end{array}$ & 42,123 & $\begin{array}{c}30.1 \\
( \pm 1.0)\end{array}$ & $4,2 \mid 4$ & $\begin{array}{c}36.7 \\
( \pm 2.9)\end{array}$ & 675 & $\begin{array}{l}29.3 \\
( \pm 1.1)\end{array}$ & 3,539 \\
\hline & Not married & $\begin{array}{c}51.9 \\
( \pm 0.4)\end{array}$ & 58,633 & $\begin{array}{c}54.9 \\
( \pm 1.1)\end{array}$ & 7,620 & $\begin{array}{c}27.9 \\
( \pm 2.7)\end{array}$ & 514 & $\begin{array}{c}58.9 \\
( \pm 1.2)\end{array}$ & 7,106 \\
\hline \multicolumn{2}{|l|}{ Lived alone } & $\begin{array}{c}* 33.7 \\
( \pm 0.4)\end{array}$ & 15,154 & $\begin{array}{l}* 39.9 \\
( \pm 1.1)\end{array}$ & 3,548 & $\begin{array}{l}* 38.5 \\
( \pm 2.9)\end{array}$ & 491 & $\begin{array}{l}* 40.1 \\
( \pm 1.1)\end{array}$ & 3,057 \\
\hline \multirow{4}{*}{ Lived in } & Nursing home & $\begin{array}{c}* 3.3 \\
( \pm 0.1)\end{array}$ & 1,475 & $\begin{array}{l}* 12.7 \\
( \pm 0.7)\end{array}$ & ।,088 & $\begin{array}{l}* 4.5 \\
( \pm 1.2)\end{array}$ & 49 & $\begin{array}{l}* \mid 4.2 \\
( \pm 0.8)\end{array}$ & 1,039 \\
\hline & Private residence & $\begin{array}{c}* 77.1 \\
( \pm 0.3)\end{array}$ & 34,659 & $\begin{array}{l}* 54.8 \\
( \pm 1.1)\end{array}$ & 4,900 & $\begin{array}{l}* 60.4 \\
( \pm 2.9)\end{array}$ & 769 & $\begin{array}{l}* 53.7 \\
( \pm 1.2)\end{array}$ & $4,13 \mid$ \\
\hline & $\begin{array}{l}\text { Private residence } \\
\left(H C^{\S}\right)\end{array}$ & $\begin{array}{l}* 11.8 \\
( \pm 0.2)\end{array}$ & 5,304 & $\begin{array}{c}* 24.7 \\
( \pm 0.9)\end{array}$ & 2,253 & $\begin{array}{l}* 23.0 \\
( \pm 2.5)\end{array}$ & 293 & $\begin{array}{c}* 25.5 \\
( \pm 1.0)\end{array}$ & 1,960 \\
\hline & $\begin{array}{l}\text { Board/assisted/ } \\
\text { group home }\end{array}$ & $\begin{array}{c}* 7.1 \\
( \pm 0.2)\end{array}$ & 3,203 & $\begin{array}{c}* 9.2 \\
( \pm 0.6)\end{array}$ & 821 & $\begin{array}{c}* 4.5 \\
( \pm 1.2)\end{array}$ & 57 & $\begin{array}{l}\text { *9.9 } \\
( \pm 0.7)\end{array}$ & 764 \\
\hline \multicolumn{10}{|c|}{ Primary Caregiver Status } \\
\hline \multicolumn{2}{|c|}{ Lives with client } & $\begin{array}{c}49.4 \\
( \pm 0.4)\end{array}$ & 54,923 & $\begin{array}{c}38.5 \\
( \pm 1.1)\end{array}$ & 5,357 & $\begin{array}{c}42.4 \\
( \pm 3.0)\end{array}$ & 779 & $\begin{array}{l}37.7 \\
( \pm 1.1)\end{array}$ & 4,554 \\
\hline \multicolumn{2}{|l|}{ Is a spouse } & $\begin{array}{l}* 29.7 \\
( \pm 0.4)\end{array}$ & 32,968 & $\begin{array}{l}* 23.6 \\
( \pm 0.9)\end{array}$ & 3,263 & $\begin{array}{c}* 34.1 \\
( \pm 2.8)\end{array}$ & 596 & $\begin{array}{l}* 22.4 \\
( \pm 1.0)\end{array}$ & 2,667 \\
\hline \multicolumn{2}{|c|}{ Is a child or child-in-law } & $\begin{array}{c}* 55.1 \\
( \pm 0.4)\end{array}$ & 61,112 & $\begin{array}{c}* 57.5 \\
( \pm 1.1)\end{array}$ & 7,899 & $\begin{array}{c}* 41.1 \\
( \pm 3.0)\end{array}$ & 719 & $\begin{array}{c}* 60.3 \\
( \pm 1.1)\end{array}$ & 7,180 \\
\hline \multicolumn{2}{|c|}{ Caregiver is unable to continue } & $\begin{array}{c}6.7 \\
( \pm 0.2)\end{array}$ & 7,456 & $\begin{array}{c}20.7 \\
( \pm 0.9)\end{array}$ & 2,919 & $\begin{array}{c}19.1 \\
( \pm 2.4)\end{array}$ & 352 & $\begin{array}{c}21.3 \\
( \pm 1.0)\end{array}$ & 2,567 \\
\hline \multicolumn{10}{|c|}{ Resource Utilization } \\
\hline \multirow{3}{*}{$\begin{array}{l}\text { Hospitalization } \\
\text { (90 days pre- } \\
\text { admission)^ }\end{array}$} & 0 & $\begin{array}{c}76.1 \\
( \pm 0.3)\end{array}$ & 86,076 & $\begin{array}{c}83.9 \\
( \pm 0.8)\end{array}$ & 11,678 & $\begin{array}{c}82.6 \\
( \pm 2.3)\end{array}$ & $|, 52|$ & $\begin{array}{c}84.1 \\
( \pm 0.9)\end{array}$ & 10,157 \\
\hline & $1-2$ & $\begin{array}{c}23.1 \\
( \pm 0.3)\end{array}$ & 26,121 & $\begin{array}{c}|5 .| \\
( \pm 0.8)\end{array}$ & 2,128 & $\begin{array}{c}16.1 \\
( \pm 2.2)\end{array}$ & 297 & $\begin{array}{c}15.2 \\
( \pm 0.8)\end{array}$ & $1,83 \mid$ \\
\hline & $\geq 3$ & $\begin{array}{c}0.8 \\
( \pm 0.1)\end{array}$ & 849 & $\begin{array}{c}1.0 \\
( \pm 0.2)\end{array}$ & 108 & $\begin{array}{c}1.3 \\
( \pm 0.7)\end{array}$ & 23 & $\begin{array}{c}0.7 \\
( \pm 0.2)\end{array}$ & 85 \\
\hline
\end{tabular}


TABLE 1. Continued

\begin{tabular}{|l|l|c|c|c|c|c|c|c|c|}
\hline $\begin{array}{l}\text { ED visits } \\
(90 \text { days pre- } \\
\text { admission) }\end{array}$ & 0 & $\begin{array}{c}82.8 \\
( \pm 0.3)\end{array}$ & 93,577 & $\begin{array}{c}80.1 \\
( \pm 0.9)\end{array}$ & 11,137 & $\begin{array}{c}81.4 \\
( \pm 2.3)\end{array}$ & 1,499 & $\begin{array}{c}79.8 \\
( \pm 0.9)\end{array}$ & 9,638 \\
\cline { 2 - 9 } & $1-2$ & $\begin{array}{c}16.0 \\
( \pm 0.3)\end{array}$ & 18,113 & $\begin{array}{c}17.7 \\
( \pm 0.8)\end{array}$ & 2,512 & $\begin{array}{c}16.1 \\
( \pm 2.2)\end{array}$ & 296 & $\begin{array}{c}18.4 \\
( \pm 0.9)\end{array}$ & 2,216 \\
\cline { 2 - 9 } & $\geq 3$ & $\begin{array}{c}1.2 \\
( \pm 0.1)\end{array}$ & 1,356 & $\begin{array}{c}2.1 \\
( \pm 0.3)\end{array}$ & 265 & $\begin{array}{c}2.5 \\
( \pm 0.9)\end{array}$ & 46 & $\begin{array}{c}1.8 \\
( \pm 0.3)\end{array}$ & 219 \\
\hline
\end{tabular}

* Missing data due to the inclusion of partial assessments

$\S$ ' $\mathrm{HC}$ ' $=$ Home care

^ Not including current hospitalization

Note: All confidence intervals are $99 \%$ (alpha $=0.01$ ) unless otherwise specified.

Within the ALC sample, the majority $(61.5 \%, 99 \%$ CI: 60.4, 62.6) did not have a primary caregiver who lived with them in their residence. ALC patients waiting for LTC who were age 75 or more were less likely to have a primary caregiver living with them, and that caregiver was more likely to be a child or child-in-law. ALC patients waiting for LTC were generally less likely to have a primary caregiver who was living with them, relative to the home care sample.

Concerning acute hospital utilization, ALC patients waiting for LTC experienced fewer recent hospitalizations, yet had a similar frequency of emergency department (ED) visits compared to the home care sample. In both samples, very few had three or more hospitalizations or ED visits during the 90 days pre-admission.

As shown in Table 2, ALC patients waiting for LTC had poorer cognitive status, diminished communication skills, more depressive symptoms, more behavioural disturbances, more functional impairment, more falls and increased health instability compared to senior home care clients. The same is true for ALC patients of advanced age ( $\geq 75$ years) versus the younger subgroup ( $<75$ years). However, there was also evidence that a subset of ALC patients awaiting LTC may still have adequate functional capacity to warrant consideration of community care options (e.g., supportive living arrangements). For example, an ALC patient in stable health condition $(72.5 \%$ of the sample), with only limited ADL impairment (39\% of the sample) or limited cognitive impairment (63.7\% of the sample) may be appropriate for transition to a community setting.

ALC patients waiting for LTC had roughly the same frequency of co-morbid conditions as home care clients, with an exception at the high end of the distribution (4+ co-morbidities). The majority of ALC patients waiting for LTC had one or more co-morbidities. Complexity in this analysis was defined by one or more of psychiatric diagnosis, signs of depression, hallucinations or delusions, cognitive impairment, any behaviour, any antipsychotic medication use, schizophrenia or bipolar disorder. A greater proportion of persons with complex health needs were found in the ALC sample than in the home care sample. ALC patients waiting for LTC who were under the age of 75 were more likely to demonstrate complexity relative to those over age 75 . 
Nonetheless, almost half the ALC patients were not complex by this definition.

As shown in Figure 1, the most common diagnosis for ALC patients waiting for LTC was arthritis, which was present in $45 \%$ (99\% CI: 43.9, 46.1) of all patients. The next most frequent diagnoses were non-Alzheimer's related dementia, stroke, diabetes and osteoporosis - each accounted for over $20 \%$ of the ALC sample. Relative to senior home care clients, ALC patients waiting for LTC had substantially higher rates of non-Alzheimer's related dementia, stroke, congestive heart failure (CHF), urinary tract infections (UTIs), psychiatric conditions, hip fracture and renal failure.

TABLE 2. Clinical characteristics

\begin{tabular}{|c|c|c|c|c|c|c|c|c|}
\hline & \multicolumn{2}{|c|}{$\begin{array}{l}\text { Home care } \\
\text { ( } \geq 65 \text { years) }\end{array}$} & \multicolumn{2}{|c|}{$\begin{array}{l}\text { ALC } \\
\text { (AII) }\end{array}$} & \multicolumn{2}{|c|}{$\begin{array}{c}\text { ALC } \\
(<75 \text { years })\end{array}$} & \multicolumn{2}{|c|}{$\begin{array}{c}\text { ALC } \\
(\geq 75 \text { years) }\end{array}$} \\
\hline & $\%(\mathrm{Cl})$ & $\mathbf{N}$ & $\%(\mathrm{Cl})$ & $\mathbf{N}$ & $\%(C l)$ & $\mathbf{N}$ & $\%(\mathrm{Cl})$ & $\mathbf{N}$ \\
\hline \multicolumn{9}{|l|}{ Cognitive Status } \\
\hline $\begin{array}{l}\text { Moderate to very severe cognitive } \\
\text { impairment' }\end{array}$ & $\begin{array}{c}\mid 1.0 \\
( \pm 0.2)\end{array}$ & 12,502 & $\begin{array}{c}36.3 \\
( \pm 1.1)\end{array}$ & 5,086 & $\begin{array}{l}32.5 \\
( \pm 2.8)\end{array}$ & 598 & $\begin{array}{l}37.2 \\
( \pm 1.1)\end{array}$ & 4,488 \\
\hline Indications of delirium (last 7 days) & $\begin{array}{c}1.9 \\
( \pm 0.1)\end{array}$ & 2,155 & $\begin{array}{c}8.6 \\
( \pm 0.6)\end{array}$ & 1,183 & $\begin{array}{c}6.3 \\
( \pm 1.5)\end{array}$ & 116 & $\begin{array}{c}8.8 \\
( \pm 0.7)\end{array}$ & 1,067 \\
\hline Impaired short-term memory & $\begin{array}{c}40.9 \\
( \pm 0.4)\end{array}$ & 46,245 & $\begin{array}{c}68.4 \\
( \pm 1.0)\end{array}$ & 9,520 & $\begin{array}{c}58.0 \\
( \pm 3.0)\end{array}$ & 1,068 & $\begin{array}{l}70.0 \\
( \pm 1.1)\end{array}$ & 8,452 \\
\hline \multicolumn{9}{|l|}{ Communication } \\
\hline Difficulty making self understood & $\begin{array}{c}8.9 \\
( \pm 0.2)\end{array}$ & 10,049 & $\begin{array}{c}25.9 \\
( \pm 1.0)\end{array}$ & 3,641 & $\begin{array}{l}24.9 \\
( \pm 2.6)\end{array}$ & 460 & $\begin{array}{c}26.4 \\
( \pm 1.0)\end{array}$ & 3,181 \\
\hline Difficulty understanding others & $\begin{array}{c}9.8 \\
( \pm 0.2)\end{array}$ & | | ,088 & $\begin{array}{c}29.5 \\
( \pm 1.0)\end{array}$ & 4,089 & $\begin{array}{c}25.7 \\
( \pm 2.6)\end{array}$ & 473 & $\begin{array}{l}29.9 \\
( \pm 1.1)\end{array}$ & 3,616 \\
\hline \multicolumn{9}{|l|}{ Mood and Behavioural Status } \\
\hline Signs of depression ${ }^{2}$ & $\begin{array}{c}13.2 \\
( \pm 0.3)\end{array}$ & 14,863 & $\begin{array}{c}17.5 \\
( \pm 0.8)\end{array}$ & 2,485 & $\begin{array}{c}21.1 \\
( \pm 2.4)\end{array}$ & 388 & $\begin{array}{c}17.4 \\
( \pm 0.9)\end{array}$ & 2,097 \\
\hline ANY behaviour ${ }^{3}$ & $\begin{array}{c}6.3 \\
( \pm 0.2)\end{array}$ & 7,161 & $\begin{array}{c}19.0 \\
( \pm 0.9)\end{array}$ & 2,622 & $\begin{array}{c}18.7 \\
( \pm 2.3)\end{array}$ & 344 & $\begin{array}{c}18.9 \\
( \pm 0.9)\end{array}$ & 2,278 \\
\hline \multicolumn{9}{|l|}{ ADL Functioning } \\
\hline Independent & $\begin{array}{c}67.1 \\
( \pm 0.4)\end{array}$ & 75,828 & $\begin{array}{c}9.1 \\
( \pm 0.6)\end{array}$ & 1,266 & $\begin{array}{c}16.3 \\
( \pm 2.2)\end{array}$ & 300 & $\begin{array}{c}8.0 \\
( \pm 0.6)\end{array}$ & 966 \\
\hline Supervision/Limited impairment & $\begin{array}{c}21.9 \\
( \pm 0.3)\end{array}$ & 24,775 & $\begin{array}{c}29.9 \\
( \pm 1.0)\end{array}$ & 4,199 & $\begin{array}{c}29.3 \\
( \pm 2.7)\end{array}$ & 540 & $\begin{array}{c}30.3 \\
( \pm 1.1)\end{array}$ & 3,659 \\
\hline Extensive assistance required & $\begin{array}{c}8.2 \\
( \pm 0.2)\end{array}$ & 9,295 & $\begin{array}{c}37.1 \\
( \pm 1.1)\end{array}$ & 5,111 & $\begin{array}{c}30.9 \\
( \pm 2.8)\end{array}$ & 569 & $\begin{array}{c}37.6 \\
( \pm 1.1)\end{array}$ & 4,542 \\
\hline Dependent & $\begin{array}{c}2.8 \\
( \pm 0.1)\end{array}$ & 3,147 & $\begin{array}{c}23.9 \\
( \pm 0.9)\end{array}$ & 3,338 & $\begin{array}{c}23.5 \\
( \pm 2.5)\end{array}$ & 432 & $\begin{array}{c}24.1 \\
( \pm 1.0)\end{array}$ & 2,906 \\
\hline
\end{tabular}


TABLE 2. Continued

\begin{tabular}{|c|c|c|c|c|c|c|c|c|c|}
\hline \multicolumn{10}{|c|}{ Health Conditions } \\
\hline \multicolumn{2}{|c|}{ Unstable condition ${ }^{4}$} & $\begin{array}{c}10.3 \\
( \pm 0.2)\end{array}$ & 11,583 & $\begin{array}{c}27.5 \\
( \pm 1.0)\end{array}$ & 3,800 & $\begin{array}{c}23.8 \\
( \pm 2.6)\end{array}$ & 438 & $\begin{array}{l}27.9 \\
( \pm 1.1)\end{array}$ & 3,362 \\
\hline \multirow[t]{2}{*}{$\begin{array}{l}\text { Falls } \\
\text { (last } 90 \text { days) }\end{array}$} & None & $\begin{array}{c}71.2 \\
( \pm 0.3)\end{array}$ & 80,276 & $\begin{array}{c}42.5 \\
( \pm 1.1)\end{array}$ & 5,940 & $\begin{array}{c}52.2 \\
( \pm 3.0)\end{array}$ & 964 & $\begin{array}{c}41.0 \\
( \pm 1.2)\end{array}$ & 4,976 \\
\hline & $\geq 2$ & $\begin{array}{c}6.7 \\
( \pm 0.2)\end{array}$ & 6,834 & $\begin{array}{c}15.7 \\
( \pm 0.8)\end{array}$ & 2,161 & $\begin{array}{c}14.3 \\
( \pm 2.1)\end{array}$ & 261 & $\begin{array}{c}16.1 \\
( \pm 0.9)\end{array}$ & 1,900 \\
\hline \multirow[t]{4}{*}{ Co-morbidities $^{5}$} & None & $\begin{array}{c}8.2 \\
( \pm 0.2)\end{array}$ & 9,241 & $\begin{array}{c}6.9 \\
( \pm 0.6)\end{array}$ & 974 & $\begin{array}{c}8.4 \\
( \pm 1.7)\end{array}$ & 155 & $\begin{array}{c}6.6 \\
( \pm 0.6)\end{array}$ & 797 \\
\hline & । & $\begin{array}{l}29.5 \\
( \pm 0.3)\end{array}$ & 33,371 & $\begin{array}{c}24.0 \\
( \pm 0.9)\end{array}$ & 3,339 & $\begin{array}{c}26.0 \\
( \pm 2.6)\end{array}$ & 479 & $\begin{array}{c}23.7 \\
( \pm 1.0)\end{array}$ & 2,864 \\
\hline & $2-3$ & $\begin{array}{c}52.5 \\
( \pm 0.4)\end{array}$ & 59,387 & $\begin{array}{c}52.4 \\
( \pm 1.1)\end{array}$ & 7,235 & $\begin{array}{c}49.3 \\
( \pm 3.0)\end{array}$ & 907 & $\begin{array}{c}52.9 \\
( \pm 1.2)\end{array}$ & 6,392 \\
\hline & $\geq 4$ & $\begin{array}{c}9.8 \\
( \pm 0.2)\end{array}$ & I I,047 & $\begin{array}{c}16.7 \\
( \pm 0.8)\end{array}$ & 2,365 & $\begin{array}{c}16.3 \\
( \pm 2.2)\end{array}$ & 300 & $\begin{array}{c}16.7 \\
( \pm 0.9)\end{array}$ & 2,021 \\
\hline \multicolumn{2}{|l|}{ Complexity $^{6}$} & $\begin{array}{c}30.0 \\
( \pm 0.4)\end{array}$ & 33,875 & $\begin{array}{c}52.4 \\
( \pm 1.1)\end{array}$ & 7,233 & $\begin{array}{c}59.6 \\
( \pm 2.9)\end{array}$ & I,098 & $\begin{array}{c}51.2 \\
( \pm 1.2)\end{array}$ & 6,181 \\
\hline
\end{tabular}

I Based on the interRAI Cognitive Performance Scale (CPS) levels $\geq 3$. Equivalent to I5 - I MMSE (Morris et al. 1994; Hartmaier et al. 1995).

${ }^{2}$ Based on the interRAI Depression Rating Scale (DRS) (Burrows et al. 2000) levels $\geq 3$.

${ }^{3}$ Includes: wandering, verbal abuse, physical abuse, socially inappropriate behaviour and resisting care.

${ }^{4}$ Based on the interRAl Changes in Health, End-stage Disease, and Signs and Symptoms (CHESS) Scale (Hirdes et al. 2003) levels $\geq 3$.

${ }^{5}$ Co-morbidities include: congestive heart failure, Alzheimer's disease, non-Alzheimer's related dementia, Parkinsonism, arthritis, osteoporosis, psychiatric diagnosis, cancer, diabetes, renal failure and COPD.

${ }^{6}$ One or more of: psychiatric diagnosis, signs of depression (DRS 3+), hallucinations/delusions, cognitive impairment (CPS $3+$ ), any behaviour, any antipsychotic medication use, schizophrenia and bipolar disorder.

Note: All confidence intervals are $99 \%(a l p h a=0.01$ ) unless otherwise specified.

FIGURE 1. Current disease diagnoses and health conditions by group

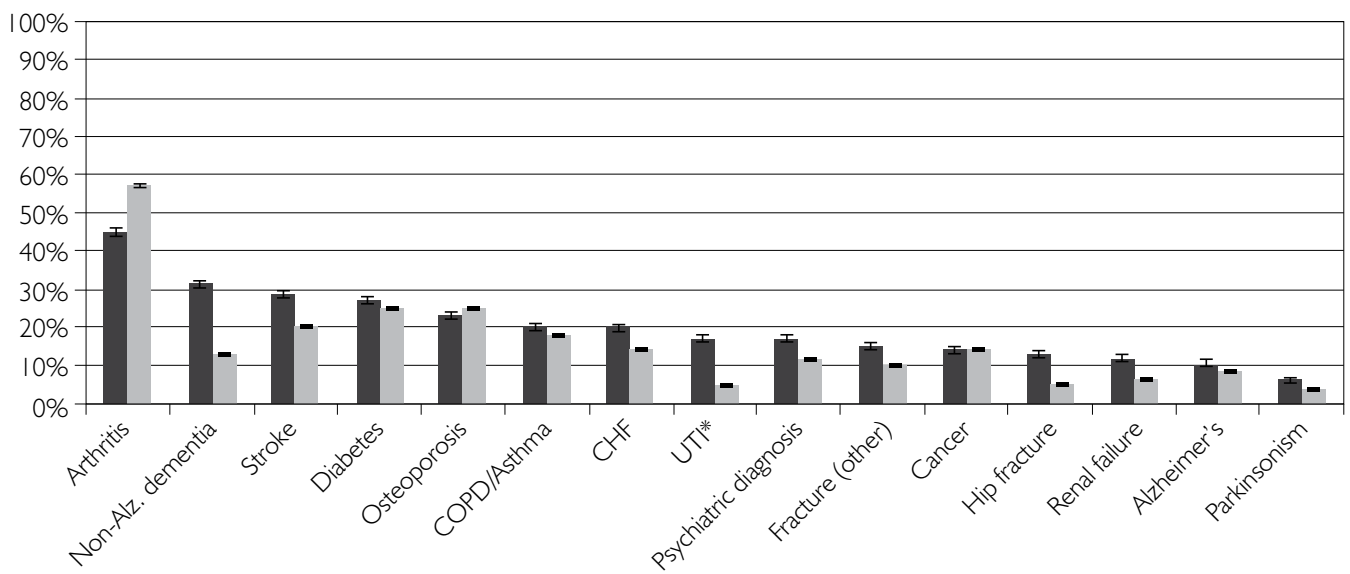

ALC Home Care

* Last 30 days

Note: All confidence intervals are $99 \%$ (alpha $=0.01$ ) unless otherwise specified. 
As shown in Figure 2, the most common needs identified by the RAI-HC's clinical assessment protocols (CAPs) among ALC patients waiting for LTC were potential to improve or prevent declines in ADLs, falls, urinary incontinence, mood and pain. Each of these CAPs was triggered for over $40 \%$ of the ALC sample. The ALC sample had substantially higher needs than the home care sample with respect to ADLs, falls, urinary incontinence, communication, medication management, bowel incontinence, cognition, behaviours, pressure ulcers and delirium.

FIGURE 2. Triggered clinical assessment protocols (CAPs)* by group

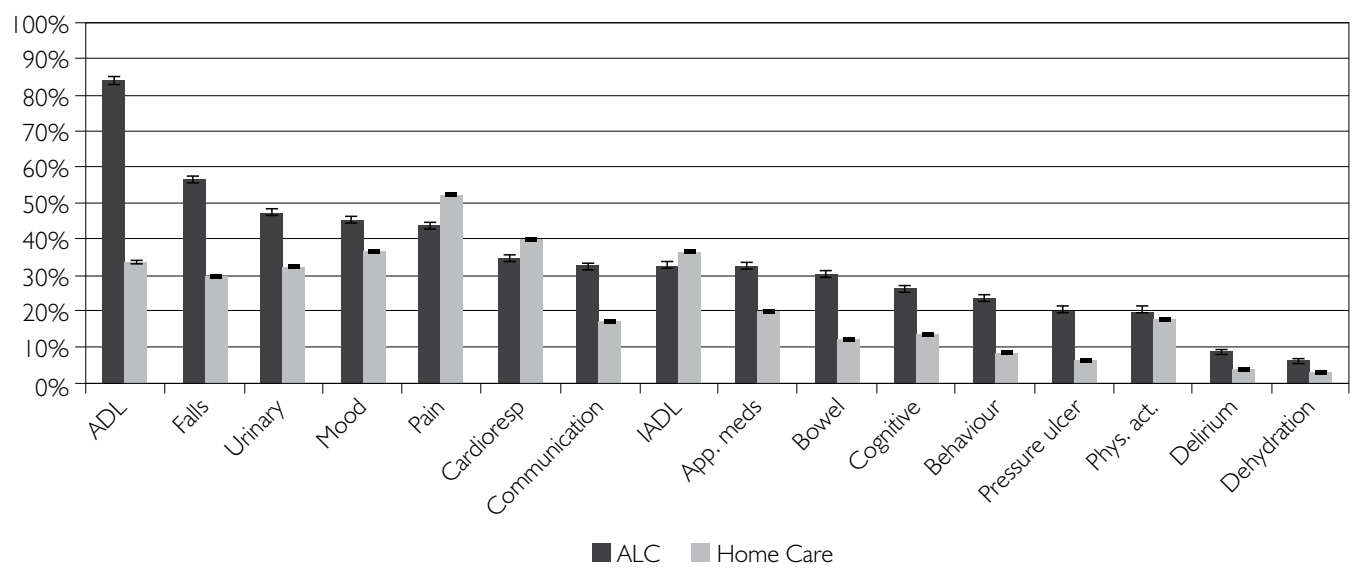

* Triggered at any level

Note: All confidence intervals are $99 \%(a$ lpha $=0.01$ ) unless otherwise specified.

\section{Discussion}

ALC patients waiting for LTC are a unique population, clinically distinct from older long-stay home care clients in Ontario. Many older adults with complex health needs receive care in multiple settings. The availability of standardized RAI-HC assessment data provides an opportunity to gain unique insights into the needs of the ALC population and compare them to the needs of existing home care clients.

Given that most community-based alternatives to LTC rely on some level of informal care capacity, access to informal support is an important consideration. ALC patients waiting for LTC were less likely than older home care clients to have a primary caregiver living with them and more likely to have a primary caregiver who expressed inability to continue. Inferring precise estimates of informal care capacity from the relationship to a primary caregiver, living situation or both can be difficult given that there may be considerable qualitative differences in caregiver capacity (Fraser and Warr 2009; Fujino and Matsuda 2009). However, the primary caregiver characteristics presented here suggest that ALC patients have less access to informal 
support compared to home care clients. This factor alone indicates that some ALC patients may be unable to return to a solitary dwelling without considerable formal home care supports.

ALC patients waiting for LTC had higher levels of functional deficits, complexity and disease burden than community-based clients. Cognitive impairment is a chief concern among functional deficits given that many community care settings require some ability on the part of residents to direct their own care. Moderate to severe cognitive impairment was found in over one-third of the ALC sample. Likewise, relative to senior home care clients, difficulties associated with stroke, dementia or acute delirium were more prevalent in ALC patients waiting for LTC. The observed rates of Alzheimer's and non-Alzheimer's related dementia are consistent with previous reports of high ALC hospitalizations and lengths of stay (CIHI 2009).

Patient well-being is an important indicator for clinicians and policy makers interested in quality of care. ALC patients waiting for LTC displayed higher levels of depression indicators compared to community-based home care clients. This finding agrees with a recent ethnographic study by Kydd (2008) that suggested older adults, termed "bed-blockers" or "delayed discharge," are anxious about their future and feel unsupported. Perhaps such anxiety is not surprising considering that a transition to an in-patient bed brings changes to daily life patterns and social consequences that are distressing to older patients. In addition, it is generally known that many older adults have negative views of residential care settings. Therefore, the transition to a less desirable care setting might have contributed to a more negative mood in the ALC sample. This factor could also have contributed to the higher number of depression indicators in the younger ALC subgroup, where the relatively early need for LTC could have had negative physiological implications.

Previous hospitalizations and ED visits were slightly less common in the ALC sample than in the home care sample. However, about $15 \%$ and $20 \%$ of ALC patients experienced one or more hospitalizations and ED visits, respectively, in the previous 90 days. Any strategy to reduce ALC days should include initiatives to prevent hospitalizations in the first place. For example, falls are a well-known risk factor for hospitalization and were experienced by $57.5 \%$ of the ALC patients compared to $28.8 \%$ of community home care clients. Likewise, the ED is an ideal setting for the use of geriatric screening to detect older persons at high risk for adverse outcomes, including ALC status. Early detection and intervention is imperative given that ALC patients admitted through the ED account for $73 \%$ of ALC bed days in Canada (Dawson et al. 2008).

Overall, the socio-demographic and clinical characteristics of the described population samples suggest that some ALC patients waiting for LTC might be successfully managed in the community with intensive and targeted home care services or with supportive living options. Moreover, a transitional care model could have an important role in returning ALC patients back to these community settings. However, this 
approach would require adequate resources and needs-based interventions in order to ensure quality care. The interRAI CAPs provide a useful starting point for planning and initiating services to support ALC patients' return to the community. Based on the current study, interventions aimed at restoring functional ability, preventing falls, managing incontinence and alleviating depressive symptoms could be of considerable benefit to a substantial portion of ALC patients in Ontario.

Despite some evidence for community-based alternatives, the real need for LTC also should not be discounted, particularly for ALC patients with extensive functional impairments. Research concerning the transitions of ALC patients to the community, the level of service intensity necessary for a successful transition, as well as cost-benefit analyses are required to determine the feasibility of community care options for ALC patients waiting for LTC admission.

\section{Strengths and limitations}

The combination of census-level records with over 300 clinical variables per record makes the RAI-HC database one of the largest data holdings available to describe ALC patients in Canada. However, given changing definitions and discharge practices for ALC in Ontario, the sample (January 2007 to September 2008) included in this analysis may not be fully representative of ALC patients waiting for LTC in coming years. Likewise, it is not known how generalizable Ontario's ALC population is to the ALC populations in other provinces.

The characteristics presented in this study reflected ALC patients' health status at the time of LTC application. Given that health status is not a static measure (particularly for hospitalized older adults), the timing of hospital RAI-HC assessment may or may not represent the characteristics of an ALC patient during his or her entire length of stay. Likewise, it is unclear whether the functional deficits and geriatric syndromes presented were reasons for, or consequences of, a prolonged ALC hospital stay.

Including the most recent, unique RAI-HC assessment in this analysis might overrepresent the last ALC episode for patients who were either assessed during multiple ALC episodes or assessed twice during the same ALC episode. However, ALC patients with more than one assessment were rare (less than 4\%). Only the most recent assessment for home care clients was used in order to eliminate a bias towards the characteristics of long-stay clients who had multiple assessments during the study period.

\section{Conclusions}

This analysis suggests that while some ALC patients waiting for LTC may be candidates for community-based care, many will require targeted services at a higher level of intensity than most community-based home care clients. ALC patients waiting for 
LTC have complex medical and psychosocial needs that would benefit from the alignment of timely need-based care decisions with access to appropriate care options.

\section{ACKNOWLEDGEMENTS}

Support for this research came from a project funded by The Change Foundation, Toronto, Ontario (www.changefoundation.ca). Dr. Hirdes is supported by a grant from the Ontario Ministry of Health and Long-Term Care (MOHLTC). Neither The Change Foundation nor the MOHLTC had responsibility for the interpretation of the data or the preparation and approval of the manuscript.

Correspondence may be directed to: Andrew P. Costa, Department of Health Studies and Gerontology, University of Waterloo, Waterloo, ON N2L 3G1; tel.: 519-888-4567, x. 37859; e-mail: acosta@uwaterloo.ca.

\section{REFERENCES}

ALC Expert Panel. 2006 (December). Appropriate Level of Care: A Patient Flow, System Integration and Capacity Solution. Report. Toronto: Ministry of Health and Long-Term Care.

Burrows, A.B., J.N. Morris, S.E. Simon, J.P. Hirdes and C. Phillips. 2000.“Development of a Minimum Data Set-Based Depression Rating Scale for Use in Nursing Homes." Age and Ageing 29(2): 165-72.

Canadian Institute for Health Information (CIHI). 2009. Alternate Level of Care in

Canada. Retrieved June 23, 2010. <http://secure.cihi.ca/cihiweb/dispPage.jsp?cw_ page $=$ PG_1751_E\&cw_topic $=17518 \mathrm{cw} \_$rel $=$AR_2610_E\#full $>$.

Covinsky, K.E., R.M. Palmer, R.H. Fortinsky, S.R. Counsell, A.L. Stewart, D. Kresevic et al. 2003. "Loss of Independence in Activities of Daily Living in Older Adults Hospitalized with Medical Illnesses: Increased Vulnerability with Age." Journal of the American Geriatrics Society 51(4): 45158.

Creditor, M.C. 1993. "Hazards of Hospitalization of the Elderly." Annals of Internal Medicine 118(3): 219-23.

Dawson, H., J. Weerasooriya and G. Webster. 2008. “Hospital Admissions via the Emergency Department: Implications for Planning and Patient Flow." Healthcare Quarterly 11(1): 20-22.

Department of Health and Wellness, Prince Edward Island. 2009. "Prince Edward Island's Healthy Aging Strategy - February 2009.” Retrieved June 23, 2010. <http://www.gov.pe.ca/publications/getpublication.php3?number $=1474>$.

Fortinsky, R.H., K.E. Covinsky, R.M. Palmer and C.S. Landefeld, 1999."Effects of Functional Status Changes Before and During Hospitalization on Nursing Home Admission of Older Adults." The Journals of Gerontology. Series A, Biological Sciences and Medical Sciences 54(10): M521-26.

Fraser, C. and D.J. Warr. 2009. “Challenging Roles: Insights into Issues for Men Caring for Family Members with Mental Illness." American Journal of Men's Health 3(1): 36-49. 
Fujino, Y. and S. Matsuda. 2009. "Prospective Study of Living Arrangement by the Ability to Receive Informal Care and Survival among Japanese Elderly" Preventive Medicine 48(1): 79-85.

Government of Alberta - Health and Wellness. 2008. "Alberta Continuing Care Strategy"

Retrieved June 23, 2010. <http://www.health.alberta.ca/initiatives/continuing-care-strategy. html>.

Graf, C. 2006. "Functional Decline in Hospitalized Older Adults." American Journal of Nursing 106(1): 58-67.

Gray, L.C., K. Berg, B.E. Fries, J.C. Henrard, J.P. Hirdes, K. Steel and J.N. Morris. 2009. “Sharing Clinical Information across Care Settings: The Birth of an Integrated Assessment System." BMC Health Services Research 29(9): 71.

Hartmaier, S.L., P.D. Sloane, H.A. Guess and G.C. Koch. 1995."Validation of the Minimum Data Set Cognitive Performance Scale: Agreement with the Mini-Mental State Examination." The Journals of Gerontology. Series A, Biological Sciences and Medical Sciences 50(2): M128-33.

Hirdes, J.P., B.E. Fries, J.N. Morris, K. Steel, V. Mor, D. Frijters, S. LaBine, C. Schalm, M.J. Stones, G. Teare, T. Smith, M. Marhaba, E. Pérez and P. Jónsson. 1999. "Integrated Health Information Systems Based on the RAI/MDS Series of Instruments." Health Care Management Forum 12(4): 30-40.

Hirdes, J.P., D.H. Frijters and G.F. Teare. 2003. “The MDS-CHESS Scale: A New Measure to Predict Mortality in Institutionalized Older People." Journal of the American Geriatrics Society 51(1): 96-100.

Hirdes, J.P., G. Ljunggren, J.N. Morris, D.H. Frijters, H. Finne-Soveri, L. Gray, M. Björkgren and R. Gilgen. 2008a. "Reliability of the interRAI Suite of Assessment Instruments: A 12-Country Study of an Integrated Health Information System." BMC Health Services Research 8: 277.

Hirdes, J.P., J.W. Poss and N. Curtin-Telegdi. 2008b. “The Method for Assigning Priority Levels (MAPLe): A New Decision-Support System for Allocating Home Care Resources.” BMC Medicine 6: 9.

interRAI Clinical Assessment Protocols (CAPs) for Use with interRAI's Community and Long-Term Care Assessment Instruments. 2008 (March). Ann Arbor, MI: interRAI.

Koehler, M., T. Rabinowitz, J.P. Hirdes, M.J. Stones, G.I. Carpenter, B.E. Fries, J.N. Morris and R.N. Jones. 2005. "Measuring Depression in Nursing Home Residents with the MDS and GDS: An Observational Psychometric Study." BMC Geriatrics 5(1): 1.

Kydd, A. 2008. "The Patient Experience of Being a Delayed Discharge." Journal of Nursing Management 16(2): 121-26.

Lim, S.C., V. Doshi, B. Castasus, J.K. Lim and K. Mamun. 2006. "Factors Causing Delay in Discharge of Elderly Patients in an Acute Care Hospital." Annals of the Academy of Medicine, Singapore 35(1): 27-32.

Martin, L., J.W. Poss, J.P. Hirdes, R.N. Jones, M.J. Stones, B.E. Fries and T. Rabinowitz. 2008. "Predictors of a New Depression Diagnosis among Older Adults Admitted to Complex Continuing Care: Implications for the Depression Rating Scale (DRS)." Age and Ageing 37(1): 51-56.

Ministry of Health and Long-Term Care (MOHLTC). 2008. "Ontario Tackles ER Waits with \$109 Million Investment." News release. Retrieved June 23, 2010. <http://www.health.gov.on.ca/ english/media/news_releases/archives/nr_08/may/nr_20080530.html >. 
Ministry of Health and Long-Term Care (MOHLTC). 2009."Public Information: Ontario's Aging at Home Strategy." Retrieved June 23, 2010. <http://www.health.gov.on.ca/english/public/ program/ltc/33_ontario_strategy.html>.

Morris, J.N., B.E. Fries, D.R. Mehr, C. Hawes, C. Phillips, V. Mor and L. Lipsitz. 1994. "MDS Cognitive Performance Scale." Journal of Gerontology 49(4): M174-82.

Morris, J.N., B.E. Fries, K. Steel, N. Ikegami, R. Bernabei, G.I. Carpenter, R. Gilgen, J.P. Hirdes and E. Topinková. 1997. "Comprehensive Clinical Assessment in Community Setting: Applicability of the MDS-HC." Journal of the American Geriatrics Society 45(8): 1017-24.

Ontario Association of Community Care Access Centres (OACCAC), Ontario Association of Non-Profit Homes and Services for Seniors, Ontario Hospital Association, Ontario Long-Term Care Association. 2006. "Alternate Level of Care - Challenges and Opportunities. A Collaborative Position Paper." Toronto: Ontario Hospital Association.

Ontario Hospital Association (OHA) ALC Survey Results. 2009 (April). Toronto: OHA. Poss, J.W., N.M. Jutan, J.P. Hirdes, B.E. Fries, J.N. Morris, G.F. Teare and K. Reidel. 2008. "A Review of Evidence on the Reliability and Validity of Minimum Data Set Data." Health Care Management Forum 21(1): 33-39.

Rock, B.D., A. Beckerman, C. Auerbach, C. Cohen, M. Goldstein and E. Quitkin. 1995.

"Management of Alternate Level of Care Patients Using a Computerized Database." Health and Social Work 20(2): 133-39.

Sager, M.A., T. Franke, S.K. Inouye, C.S. Landefeld, T.M. Morgan, M.A. Rudberg et al. 1996. "Functional Outcomes of Acute Medical Illness and Hospitalization in Older Persons." Archives of Internal Medicine 156(6): 645-52.

\section{HealthcarePolicy.net}

